\title{
Violencia de género en mujeres con consumo de sustancias ingresadas en el hospital general: cribado y prevalencia
}

\section{Intimate partner violence among female drug users admitted to the general hospital: screening and prevalence}

\author{
Clara Caldentey*,*****; Judit Tirado-Muñoz**; Tessie Ferrer***; Francina Fonseca**,****; \\ Paola Rossi**,****; Juan Ignacio Mestre-Pintó**; Marta Torrens*,**,****. \\ *Universitat Pompeu Fabra-Universitat Autònoma de Barcelona, Barcelona, Spain; **Addiction Research Group, IMIM-Institut \\ Hospital del Mar d'Investigacions Mèdiques; ***Complejo Hospitalario Universitario de Granada, Hospital Universitario San \\ Cecilio, Granada, Spain; ****Institute of Neuropsychiatry and Addictions, Parc de Salut Mar, Barcelona, Spain; *****Servicio \\ de Psiquiatría, Consorci Hospitalari de Vic, España.
}

\section{Resumen}

La violencia de género (VG) es un problema de salud pública a escala mundial. Existen determinados factores asociados a un aumento de la prevalencia, como el consumo de sustancias. Se realizó un estudio transversal con el objetivo de determinar la prevalencia de VG en las mujeres que ingresaron en el Hospital del Mar (Barcelona) por cualquier motivo médico/quirúrgico y con el diagnóstico de trastorno por consumo de sustancias. Secundariamente, se pretendió validar la versión en español del cuestionario Hurt, Insulted, Threatened with Harm, Screamed (HITS). Se evaluaron a todas las pacientes mediante dos cuestionarios de detección de VG, el Composite Abuse Scale (CAS) y el HITS. De las 52 pacientes entrevistadas durante el período de estudio, 46 respondieron ambos cuestionarios. Según el cuestionario CAS, 23 pacientes $(50 \%)$ presentaron VG alguna vez en la vida y $11(23,9 \%)$ en el último año. El consumo de cannabis se asoció a una mayor gravedad de la VG (IC95\% 3,5-28,9, $p=.013)$. La prevalencia de VG, según el HITS, fue de $39,1 \%$ (18 pacientes) en los últimos 12 meses. El HITS mostró en relación al CAS una especificidad del 100\% y una sensibilidad del $78 \%$. Un punto de corte $\mathrm{x} \in[6,7]$, obtenido mediante el análisis ROC, fue el que mejor discriminó al 91\% de las víctimas y al $100 \%$ de las no-víctimas. Los resultados obtenidos demostraron una elevada prevalencia de VG entre las mujeres consumidoras de más de una sustancia de abuso. Por ese motivo, se recomienda incorporar el cribado sistemático mediante el cuestionario HITS

Palabras clave: Psiquiatría de enlace; Violencia de género; Cribado; Trastorno por consumo de sustancias; Validación.

\begin{abstract}
Intimate partner violence (IPV) is a public health problem worldwide. Several factors have been found to be associated with an increased prevalence of IPV, such as substance use. A cross-sectional study was conducted with the aim of determining the prevalence of IPV among women entering Hospital del Mar (Barcelona) for any medical/ surgical reason, and who had a diagnosis of substance use disorder. Secondly, it was intended to psychometrically validate the Spanish version of the Hurt, Insulted, Threatened with Harm, Screamed (HITS) questionnaire. All patients were assessed by two IPV questionnaires, the Composite Abuse Scale (CAS) and HITS. Out of 52 patients interviewed, 46 answered both questionnaires. According to the CAS questionnaire, 23 patients (50\%) experienced IPV at some point in their lives and 11 (23.9\%) in the last year. Cannabis consumption was also associated with an increased severity of IPV (95\% CI 3.5-28.9, $p$ $=.013)$.According to the HITS questionnaire, there was a prevalence of $39.1 \%$ (18 patients) in the last 12 months. HITS had a specificity of $100 \%$ and a sensitivity of $78 \%$ relative to the CAS questionnaire A cut-off score $\mathrm{x} \in$ [6.7], derived through ROC analysis, correctly discriminated $91 \%$ of the victims and $100 \%$ of the non-victims. The results obtained showed that the prevalence of IPV was very high among women who suffered from more than one substance use disorder. Therefore, it is highly recommended to systematically screen for IPV victimization by putting the HITS questionnaire into practice. Key Words: Liaison psychiatry; Intimate partner violence; Screening test; Substance use disorder; Validation.
\end{abstract}




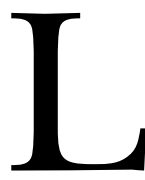

a violencia de género (VG) es un tema relevante en salud pública debido a sus consecuencias negativas y su elevada prevalencia. El concepto de VG incluye la violencia o amenaza tanto física, sexual o psicológica por parte de una persona hacia su pareja actual o anterior, sean o no del mismo sexo (WHO, 2013). No obstante, en lo que se refiere a este estudio, se utilizó el término VG como violencia hacia la mujer. Aunque algunos estudios demuestran prevalencias similares de victimización y perpetración, la violencia hacia las mujeres da lugar a consecuencias más graves (p.ej. muerte) (Desmarais, Reeves, Nicholls, Telford y Fiebert, 2012; Langhinrichsen-Rohling, McCullars y Misra, 2012). Según Devries et al. (2013), a nivel global, el 30\% de las mujeres han experimentado violencia física y/o sexual por parte de sus parejas en algún momento de sus vidas. En España, el 12.5\% de las mujeres mayores de 16 años dicen haber sufrido violencia física o sexual por parte de sus parejas o exparejas a lo largo de su vida (Ministerio de Sanidad y Políticas Sociales, 2015).

En una revisión sistemática de los estudios realizados sobre los factores de riesgo asociados a la VG, se observó que los antecedentes de violencia en la familia o abuso en la infancia, pertenecer a una minoría étnica, tener escasos ingresos económicos, o un cociente intelectual bajo, se asociaban a padecer VG (Capaldi, Knoble, Shortt y Kim, 2012). Las consecuencias de la VG pueden llegar a ser muy importantes y derivar en lesiones y problemas de salud mental graves en las víctimas y los niños testimonios de VG. Las víctimas de VG tienen más riesgo de sufrir patologías ginecológicas (incluidas infecciones de transmisión sexual y patología del embarazo), gastrointestinales, dolor agudo/crónico y de cometer suicidio respecto a las mujeres que no son víctimas de VG (Campbell, 2002; Hussain et al., 2015). Asimismo, algunas patologías mentales como la depresión, los trastornos de ansiedad, el trastorno de estrés postraumático y el trastorno por consumo de sustancias se han asociado a la VG (Gilchrist, Blázquez y Torrens, 2012; Reingle, Jennings, Connell, Businelle y Chartier, 2014). Además, más de una tercera parte de los homicidios hacia mujeres son realizados por parte de sus parejas (Stöckl et al., 2013). Así pues, la VG implica, además de los problemas legales y judiciales, problemas sanitarios graves. En este sentido, desde el punto de vista de la salud, el personal sanitario tiene un papel importante en la prevención y detección de la VG.

$\mathrm{Si}$ bien numerosos estudios indican que existe una significativa y mayor prevalencia (25-75\%) de todos los tipos de violencia y de la gravedad de ésta entre las mujeres víctimas adictas al alcohol u otras sustancias (El-Bassel, Gilbert, Witte, Wu y Chang, 2011; Feingold, Washburn, Tiberio y Capaldi, 2015; Weaver, Gilbert, El-Bassel, Resnick y Noursi, 2015), no se han realizado estudios en nuestro medio sobre la prevalencia de VG en las mujeres consumidoras de sustancias ingresadas en servicios hospitalarios. Así, el objetivo de este estudio fue doble: 1) detectar la prevalencia de VG en mujeres con trastorno por uso de sustancias que fueron ingresadas en un hospital general por cualquier patología relacionada o no con su adicción, 2) validar en mujeres consumidoras de sustancias la versión española del cuestionario Hurt, Insulted, Threatened with Harm and Screamed (HITS) (Sherin, Sinacore, Li, Zitter y Shakil, 1998), una entrevista de cribado de VG que requiere muy poco tiempo de administración y que puede ser útil en la clínica diaria.

\section{Método}

\section{Participantes}

Se evaluaron todas las mujeres que durante los periodos de noviembre 2013-febrero 2014 ( $\mathrm{n}=18)$, mayo-junio 2014 ( $\mathrm{n}=3$ ) y septiembre 2014-febrero 2015 ( $n=25)$, recibieron atención por parte del equipo de psiquiatría de enlace (interconsultas de adicciones) del Instituto de Neuropsiquiatría y Adicciones (INAD) del Parc de Salut Mar de Barcelona, o ingresaron en la unidad de desintoxicación del Hospital del Mar.

Los criterios de inclusión del estudio fueron: 1) haber tenido pareja sentimental en algún momento de la vida, 2) ser mayor de 18 años, 3) estar diagnosticada de Trastorno por consumo de sustancias (DSM-5) con o sin otra patología médico-quirúrgica asociada. Fueron criterios de exclusión la presencia de: 1) trastornos cognitivos graves, 2) intoxicación grave o síndrome de abstinencia y 3) barrera idiomática, en el momento de la evaluación. Todas aquellas mujeres que cumplían los criterios de inclusión fueron informadas de las características del estudio y de la confidencialidad en que los datos iban a ser tratados y se les pidió que firmaran el consentimiento informado para poder ser incluidas en el estudio. El estudio fue aprobado por el Comité Ético de Investigación Clínica del Parc de Salut Mar (CEIC-PSMAR).

En el período estudiado ingresaron 52 pacientes, de las cuales $4(7.7 \%)$ no aceptaron participar en el estudio (porcentaje de respuesta: $92.3 \%$ ) y 2 fueron excluidas por presentar un trastorno cognitivo severo. De las 46 pacientes estudiadas, 24 (52.2\%) estaban ingresadas en la unidad de desintoxicación mientras que $22(47.8 \%)$ procedían de la interconsulta hospitalaria.

No se encontraron diferencias significativas en las características sociodemográficas ni clínicas de la muestra en función del periodo de reclutamiento.

\section{Instrumentos de medida}

A todas las participantes se les administró un cuestionario ad hoc de datos socio-demográficos y clínicos (edad, sexo, estado civil, procedencia, situación laboral, años de escolaridad, domicilio, entorno social consumidor, antecedentes legales, antecedentes familiares de adicciones o de trastornos psiquiátricos, así como las características del consumo de sustancias, motivo de ingreso u otros diagnósticos), por parte del equipo habitual de interconsultas hospitalarias del Proceso de Adicciones-INAD. 
La evaluación de la VG se realizó mediante la versión castellana del cuestionario "Composite Abuse Scale" (CAS) (Tirado-Muñoz, Gilchrist, Lligoña y Torrens, 2015). Las participantes debían contestar la frecuencia con la que ocurrieron los hechos en el último año en la relación de pareja actual o la última previa. El cuestionario CAS se compone de un total de 30 preguntas que se clasifican en 4 subescalas: violencia combinada severa (8 ítems, 0-40 puntos), abuso físico (7 ítems, 0-35 puntos), abuso emocional (11 ítems, 0-55 puntos) y acoso/amenaza (4 ítems, 0-20 puntos). Las preguntas se responden según la frecuencia en la que ocurrió el acontecimiento: nunca ( 0 puntos), sólo una vez (1 punto), varias veces ( 2 puntos), una vez al mes ( 3 puntos), una vez a la semana (4 puntos), a diario ( 5 puntos). El tiempo de administración es de 15-20 minutos aproximadamente. Una puntuación total $\geq 7$ puntos indica VG, siendo las puntuaciones más altas relacionadas con la gravedad de la violencia. Además, dentro de las subescalas de los distintos tipos de violencia, los puntos de corte empleados son: violencia grave combinada (1 punto), abuso físico (1 punto), abuso emocional (3 puntos) y acoso/amenaza (2 puntos). Este cuestionario demostró una buena consistencia interna (alfa de Cronbach $>0.85$ ) para las 4 subescalas y las correlaciones ítem-total corregidas fueron >0.5 (Hegarty, Sheehan y Schonfeld, 1999).

El cuestionario HITS (Sherin et al., 1998) se compone de 4 preguntas: (1) “¿con qué frecuencia su pareja le provoca daño físico?”, (2) “¿con qué frecuencia su pareja le insulta o le habla con un tono paternalista”?, (3) “¿con qué frecuencia le amenaza con que le va a hacer daño?”, (4) “¿con qué frecuencia su pareja le grita o le dice palabrotas?”. Éstas se responden según la frecuencia con la que se produjo la situación, desde nunca (1 punto) a con frecuencia (5 puntos). La puntuación total puede variar en un rango de 4 a 20 puntos y 10 o más puntos se considera víctima de VG. El tiempo de administración es de 1 minuto.

A diferencia del CAS, el HITS sólo evalúa VG en la pareja actual. Este cuestionario fue traducido al castellano mediante un proceso de traducción y retrotraducción. La versión castellana no está validada en mujeres consumidoras de sustancias. Los cuestionarios CAS y HITS fueron administrados por un investigador independiente del equipo habitual de interconsultas de adicciones.

\section{Procedimiento}

El equipo de interconsultas de adicciones recibe de forma diaria solicitudes de valoración e intervención de pacientes ingresadas en el Hospital del Mar que presentan de forma concomitante un trastorno por consumo de sustancias. Dentro de los procedimientos habituales en la interconsulta se recogen los datos sociodemográficos y clínicos y las características del consumo de sustancias mediante un cuestionario ad hoc anteriormente ya mencionado. Cuando se realizaba una intervención en una paciente que cumplía los criterios de inclusión se informaba a las investigadoras independientes para completar la recogida de información para el estudio. En caso de que durante la entrevista, la paciente, víctima de VG, solicitase ayuda se le explicaba el circuito de ayuda establecido por el hospital y se comunicaba la situación a la trabajadora social correspondiente para acceder a los recursos de atención a las víctimas.

\section{Análisis de datos}

Se llevó a cabo un análisis descriptivo de la muestra. Se utilizaron pruebas de Chi cuadrado y el test exacto de Fisher para las variables cualitativas y la prueba T de Student para las cuantitativas. Se dividió la muestra en función de haber experimentado o no violencia según CAS. A continuación, se calculó el coeficiente kappa de Cohen para determinar el grado de concordancia entre los dos cuestionarios así como la sensibilidad, especificidad, el valor predictivo positivo (VPP) y el valor predictivo negativo (VPN) del cuestionario HITS respecto al CAS como patrón oro. Finalmente, se realizó el análisis de la curva característica de operación del receptor (ROC) para determinar el punto de corte de la escala HITS en el que se alcanzaba la sensibilidad y especificidad más alta y evaluar también la capacidad discriminativa del HITS respecto al CAS. Para valorar los resultados ser eligió un nivel de significación de $p<.05$.Todos los análisis univariantes y bivariantes se realizaron mediante el programa informático SPSS, versión 20.

\section{Resultados}

\section{Características socio-demográficas y clínicas}

La media de edad fue de 46.6 años (DT=10.6). Veinticinco pacientes $(54.3 \%)$ estaban casadas o emparejadas (10 solteras, 9 separadas y 2 viudas). En cuanto a las características laborales de éstas, un $23.9 \%$ estaban en paro, un 23.9\% recibían una pensión diferente a la de invalidez, y solamente un $17.4 \%$ eran trabajadoras activas. En la Tabla 1 se describen detalladamente las características socio-demográficas y clínicas de la muestra. Los diagnósticos de ingreso más frecuentes en medio hospitalario fueron: trastorno por consumo de alcohol $(n=10,21.7 \%)$ y trastorno por consumo de cocaína $(n=7,15.2 \%)$. La hepatopatía crónica fue un diagnóstico secundario también presente en un $35.6 \%$ de las participantes así como el trastorno de la personalidad, $27.3 \%$ (véase Tabla 1).

\section{Historia toxicológica}

La mayoría de las pacientes $(\mathrm{n}=36,78.3 \%)$ consumían alcohol, que además, era la droga principal de consumo en $24(52.2 \%)$ casos. Las otras sustancias más consumidas, a parte de la nicotina, fueron la cocaína $(43.5 \%)$ y el cannabis $(30.4 \%)$, siendo la cocaína la droga principal en 7 casos (15.2\%) (véase Tabla 1 y 2 ). La mayoría de las participantes eran policonsumidoras, siendo la media de número de sustancias consumidas de $2.9(D T=1.7)$. En la tabla 2 se muestra 
Tabla 1. Características socio-demográficas y clínicas de la muestra

\begin{tabular}{|c|c|c|c|c|}
\hline & Participantes & & & \multirow{3}{*}{$p$} \\
\hline & $N=46$ & $S I(n=23)$ & NO $(n=23)$ & \\
\hline & $n(\%)$ & n (\%) & n (\%) & \\
\hline \multicolumn{5}{|l|}{ Socio-demográficos } \\
\hline Edad [media \pm DT] (años) & $46.6 \pm 10.6$ & $43.70 \pm 10$ & $49.48 \pm 10.6$ & 0.06 \\
\hline Estado civil & & & & $0.63^{*}$ \\
\hline Casado & $25(54.3)$ & $12(52.2)$ & $13(56.5)$ & \\
\hline Procedencia & & & & $1^{*}$ \\
\hline Extranjero & $6(13)$ & $3(13)$ & $3(13)$ & \\
\hline Situación laboral & & & & $0.59^{*}$ \\
\hline Desempleo o pensión & $22(47.8)$ & $12(52.2)$ & $10(43.5)$ & \\
\hline Escolaridad años & $10.6 \pm 4.4$ & $11.14 \pm 4.7$ & $10.17 \pm 4.1$ & 0.47 \\
\hline Entorno social consumidor & $22(48.9)$ & $12(54.5)$ & $10(43.5)$ & 0.46 \\
\hline Antecedentes legales & $11(23.9)$ & $6(26.1)$ & $5(22.7)$ & $0.56^{*}$ \\
\hline Ant. fam.* de adicción & $26(60)$ & $14(63.6)$ & $12(54.5)$ & 0.54 \\
\hline Ant. fam. psiquiátricos & $10(22.7)$ & $4(18.2)$ & $6(27.3)$ & 0.47 \\
\hline Lugar de ingreso & & & & 0.24 \\
\hline Unidad de Desintoxicación & $24(52.2)$ & $14(60.9)$ & $10(43.5)$ & \\
\hline Interconsulta & $22(47.8)$ & $9(39.1)$ & $13(56.5)$ & \\
\hline \multicolumn{5}{|l|}{ Otros diagnósticos } \\
\hline Trastorno depresivo & $11(24.4)$ & $5(22.7)$ & $6(26.1)$ & 0.79 \\
\hline Trastorno de la personalidad & $12(27.3)$ & $8(38.1)$ & $4(17.4)$ & 0.12 \\
\hline Infección por VIH & $10(22.2)$ & $7(31.8)$ & $3(13)$ & $0.16^{*}$ \\
\hline Infección por VHC & $10(21.7)$ & $8(34.8)$ & $2(8.7)$ & $0.09^{*}$ \\
\hline Hepatopatía crónica & $16(35.6)$ & $6(27.3)$ & $10(43.5)$ & $0.13^{*}$ \\
\hline \multicolumn{5}{|l|}{ Droga principal } \\
\hline Heroína & $7(15.2)$ & $4(17.4)$ & $3(13.0)$ & $1.00^{*}$ \\
\hline Alcohol & $24(52.2)$ & $9(39.1)$ & $15(65.2)$ & 0.07 \\
\hline Nicotina & $1(2.2)$ & $1(4.3)$ & $0(0)$ & $1.00^{*}$ \\
\hline Hipnosedantes & $3(6.5)$ & $2(8.7)$ & $1(4.3)$ & $1.00^{*}$ \\
\hline Cocaína & $7(15.2)$ & $5(21.7)$ & $2(8.7)$ & $0.41^{\star}$ \\
\hline Cannabis & $2(4.3)$ & $1(4.3)$ & $1(4.3)$ & $1.00^{*}$ \\
\hline
\end{tabular}

Nota. VG: Violencia de género; CAS: Composite Abuse Scale; DT: desviación típica; Ant. Fam.: antecedentes familiares; VIH: Virus Immunodeficiencia Humana; VHC: Virus Hepatitis C. *Test exacto de Fisher.

además las características del consumo de sustancias (edad de inicio, periodo de abstinencia máximo y consumo en los últimos 30 días).

\section{Prevalencia de VG según la escala CAS}

De acuerdo con el CAS, 23 pacientes $(50 \%)$ presentaron VG alguna vez en la vida siendo, y un $23.9 \%$ en el último año. De éstas, 16 pacientes $(34.8 \%)$ padecieron abuso combinado grave, $21(45.7 \%)$ abuso físico, 22 (47.8\%) abuso emocional y por último, 16 mujeres $(34.8 \%)$ sufrieron acoso. No se encontraron diferencias significativas en las ca- racterísticas sociodemográficas ni clínicas de la muestra en función de haber experimentado o no VG en el último año, aunque un mayor número de mujeres víctimas de violencia eran VHC positivas. No se encontraron diferencias significativas en la victimización de VG para ninguna de las drogas principales (véase Tabla 1).

Las sustancias de abuso que más se asociaron a la presencia de VG fueron el cannabis $(\mathrm{p}=.01)$ y la cocaína $(\mathrm{p}=$ .02 ), siendo las mujeres consumidoras de cannabis las que obtuvieron una mayor puntuación en el cuestionario CAS (media 26.93) (IC95\% 3.5-28.9, p = .013) (véase Tabla 2). 
Tabla 2. Prevalencia de VG y características del consumo según sustancias consumidas

\begin{tabular}{|c|c|c|c|c|c|c|c|c|c|c|c|}
\hline & \multirow{2}{*}{$\begin{array}{c}\text { Participantes } \\
\mathrm{N}=46 \\
\end{array}$} & \multicolumn{2}{|c|}{ VG CAS } & \multicolumn{3}{|c|}{$\begin{array}{l}\text { Edad media } \\
\text { inicioconsumo }\end{array}$} & \multicolumn{2}{|c|}{$\begin{array}{l}\text { Período máximo de } \\
\text { abstinencia (meses) }\end{array}$} & \multicolumn{2}{|c|}{$\begin{array}{l}\text { Días de consumo } \\
\text { en el último mes }\end{array}$} & \multirow{3}{*}{$\begin{array}{c}\text { CAS* } \\
\text { puntuación } \\
\text { media }\end{array}$} \\
\hline & & Sí $(n=23)$ & NO $(n=23)$ & \multirow{2}{*}{ P } & \multirow{2}{*}{ Media } & \multirow{2}{*}{ DT } & \multirow{2}{*}{ Media } & \multirow{2}{*}{ DT } & \multirow{2}{*}{ Media } & \multirow{2}{*}{ DT } & \\
\hline & $\mathrm{n}(\%)$ & n (\%) & n (\%) & & & & & & & & \\
\hline \multicolumn{12}{|l|}{$\begin{array}{l}\text { Sustancias } \\
\text { consumidas }\end{array}$} \\
\hline Heroína & $14(30.4)$ & $10(43.5)$ & $4(17.4)$ & 0.06 & 22.8 & 8.3 & 76.5 & 99.3 & 9.5 & 13.7 & 24.93 \\
\hline Alcohol & $36(78.3)$ & 17 (73.9) & $19(82.6)$ & 0.47 & 17.44 & 5.2 & 9.33 & 18.9 & 19.5 & 12.7 & 14.75 \\
\hline Nicotina & 34 (73.9) & $19(82.6)$ & $15(65.2)$ & 0.18 & 15.32 & 3.5 & 14.16 & 58.1 & 19.8 & 14.2 & 17.68 \\
\hline Hipnosedantes & $12(26.1)$ & $7(30.4)$ & $5(21.7)$ & 0.5 & 25.75 & 9.3 & 0.17 & 0.6 & 25.8 & 10 & 19.17 \\
\hline Cocaína & $20(43.5)$ & $14(60.9)$ & $6(26.1)$ & $0.02^{\star}$ & 21.8 & 6.5 & 43.6 & 56.9 & 14.6 & 15.0 & 24.85 \\
\hline Cannabis & $14(30.4)$ & $11(47.8)$ & $3(13)$ & 0.01 ** & 17.5 & 7.5 & 14 & 22.1 & 17.5 & 15.0 & 26.93 \\
\hline
\end{tabular}

Nota. VG: Violencia de género; CAS: Composite Abuse Scale; DT: desviación típica. ${ }^{\star p}$ ২.05 **p<0.01

\section{Validación del cuestionario HITS}

Según el HITS, la prevalencia de la VG fue de $39.1 \%$ (18 pacientes) en los últimos 12 meses. En este estudio, el cuestionario HITS mostró en relación al CAS una especificidad del $100 \%$ y una sensibilidad del $78 \%$ (5 pacientes que según el CAS sufrieron VG, no se detectaron en el cuestionario HITS). Además, presentó un VPP del $100 \%$ y un VPN del $82 \%$. El coeficiente kappa de Cohen obtenido fue de 0.78 $(p=.000)$, por lo tanto, los dos cuestionarios son concordantes. Del análisis de la curva ROC para el HITS respecto al CAS se obtuvo un área debajo de la curva $(A U C)$ de 0.97 (IC 95\% [0.92-1], $p=.000)$. Un punto de corte $\mathrm{x} \in$ [6.7] fue el que maximizó mejor la sensibilidad y la especificidad, discriminando correctamente al $91 \%$ de las víctimas y al 100\% de las no-víctimas (véase Figura 1).

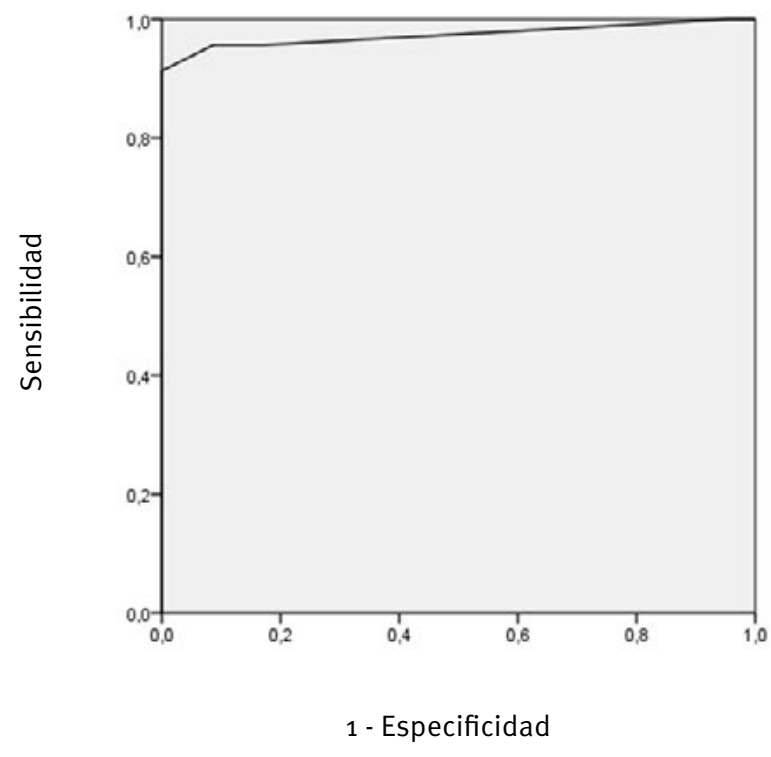

Figura 1. Curva ROC para la detección de la VG del cuestionario Hurt, Insulted, Threatened with harm, and Screamed at (HITS) en relación al Composite Abuse Scale (CAS).

\section{Discusión}

La presencia de VG en las mujeres con trastorno por consumo de sustancias atendidas en psiquiatría de enlace (interconsulta de adiciones) que ingresaron en servicios hospitalarios, es elevada. El 50\% de las pacientes entrevistadas en este estudio habían experimentado VG a lo largo de la vida, y un $23.9 \%$ en el último año. Este porcentaje obtenido es similar al de otros estudios, en los que se estima una prevalencia de entre el 25-75\% entre las mujeres consumidoras de sustancias (El-Bassel et al., 2011; Gilchrist et al., 2012), claramente elevado en comparación a la población general, en la que es del $12.5 \%$ en España (Ministerio de Sanidad y Políticas Sociales, 2015).

En relación a los trastornos por consumo de sustancias que más se asocian a padecer VG, el consumo de alcohol se ha relacionado estrechamente con la VG (Devries et al., 2014; Kraanen, Vedel, Scholing y Emmelkamp, 2014); no obstante, los trastornos por uso de alcohol y cocaína son considerados los más predictores para convertirse en víctima de VG entre las mujeres consumidoras (Kraanen et al., 2014). Además, según los resultados de la National Epidemiologic Survey on Alcohol and Related Conditions (NESARC), los trastornos por consumo de opioides y cannabis también se asocian a experimentar VG (Reingle et al., 2014; Smith, Homish, Leonard y Cornelius, 2012).

Un metánalisis reciente sobre estudios longitudinales ha indicado la existencia de una relación bidireccional entre el consumo de alcohol y la VG (OR: 1.80, IC 95\% [1.58-2.06]) (Devries et al., 2014), también mostrándose esta asociación, aunque no de manera significativa en el presente estudio. La ausencia de estudios longitudinales que estudien esta asociación no ha permitido establecer causalidad para otros consumos de sustancias como tampoco discriminar si esta asociación es siempre bidireccional.

Existen pocos estudios en los que se controle el factor de confusión de que la pareja fuese consumidora de alcohol. En un estudio estadounidense se ha demostrado que 
las parejas que poseen un mismo patrón de consumo tenían menor probabilidad de padecer violencia de género. Por lo tanto, el hábito de consumo es un factor predictor de VG, dando a entrever que los conflictos en la pareja son más predictivos de VG que el propio consumo de alcohol (Leadley, Clark y Caetano, 2000).

Se han propuesto diferentes mecanismos para explicar la relación ente los trastornos por consumo de sustancias y ser víctima de VG. Algunos han propuesto que los problemas relacionados con el consumo conducen a una situación de estrés en la relación de pareja que desemboca en la VG; otros, en cambio, hipotetizan que las víctimas de violencia de género consumen sustancias para hacer frente al estrés, la ansiedad y el dolor que genera sufrir VG (Kraanen et al., 2014). Entre las mujeres en programa de mantenimiento con metadona, el consumo de sustancias puede representar una forma de automedicación para poder hacer frente a las consecuencias negativas de la VG experimentada (El-Bassel, Gilbert, Wu, Go y Hill, 2005).

La VG entre las mujeres consumidoras de sustancias se asocia a problemas de salud mental (Cohen, Field, Campbell y Hien, 2013; Gilchrist et al., 2012); puede aumentar el riesgo de contagio de enfermedades como el VIH o VHC a través de conductas sexuales y de inyección no seguras (Wagner et al., 2009). Dadas las consecuencias devastadoras que la VG provoca en la salud física y mental de la mujer, la necesidad de detectar VG se considera crucial incluso en aquellas mujeres que no buscan tratamiento por el uso de sustancias pero que son atendidas en medios hospitalarios.

Otro hallazgo importante de este estudio es la asociación, aunque no significativa, entre ser víctima de VG en la pareja y el VHC, siendo consistente en estudios previos donde se ha evaluado la prevalencia de VHC en mujeres consumidoras de sustancias (Gilbert et al., 2000) y se ha encontrado una fuerte asociación entre ser VHC + y haber experimentado VG. Esto debería tenerse en cuenta a la hora prescribir nuevas opciones de tratamiento entre las mujeres consumidoras de sustancias ya que la posibilidad de reinfección del VHC es elevada entre las mujeres víctimas de VG.

Puesto que no se disponen de instrumentos de cribado de VG en español, el hallazgo de que la versión española del HITS es una herramienta útil para el cribado de VG en esta población es muy relevante. El HITS presenta un tiempo de administración mucho más breve que el CAS, por lo que es una buena alternativa para ser usado en la detección de VG en entornos sanitarios. En un estudio que se realizó para estudiar la validez y fiabilidad del HITS en población hispana, se obtuvieron resultados similares a los nuestros. El punto de corte de 5.5 fue el que mejor discriminó a las mujeres víctimas de VG (Chen, Rovi, Vega, Jacobs y Johnson, 2005). No obstante, se comparaba el HITS con respecto al WAST en versión española, otro cuestionario utilizado para la detección de la VG. Cabe decir, por último, que se necesitan más estudios para comprobar la consistencia interna y valorar la modificación del punto de corte para conseguir una mejor capacidad discriminativa del HITS.

En conclusión, los resultados obtenidos en este estudio confirman que las mujeres que consumen sustancias son una población de elevado riesgo de padecer VG en nuestro medio. Por ello, se recomienda el cribado sistemático mediante el cuestionario HITS en todas las mujeres con el diagnóstico de más de un trastorno por consumo de sustancias que ingresan en medio hospitalario independientemente de cuál sea el motivo de ingreso. La detección del consumo de sustancias como un factor de riesgo modificable debería ser un punto de partida para dirigir futuras estrategias de intervención y políticas de prevención de la VG. En este sentido, se dispone de una intervención cognitivo-conductual manualizada que ha mostrado eficacia en la reducción de la VG entre mujeres consumidoras de sustancias (Tirado-Munoz et al., 2015).

Finalmente, se deben mencionar las diversas limitaciones del estudio. Por un lado, el número de mujeres que necesitaron atención por parte del equipo de interconsultas fue reducido. A pesar de esto, el porcentaje de no respuesta de la muestra fue del $7.7 \%$, siendo éste menor en comparación con otros estudios realizados en mujeres con trastorno por consumo de sustancias y VG (Kraanen et al., 2014). Debido al reducido tamaño muestral, los resultados de este estudio deben ser interpretados con cautela y no deberían ser extrapolados a poblaciones de otras áreas geográficas.

El estudio se llevó a cabo en diferentes periodos temporales por disponibilidad de los investigadores, no pudiendo descartar la presencia de un posible sesgo temporal.

\section{Agradecimientos}

Este trabajo fue parcialmente financiado por el proyecto :RD12/0028/009 del Fondo de Investigaciones Sanitarias, Instituto Carlos III-FEDER y la AGAUR (2014 SGR790). Todos los autores contribuyeron igualmente a este trabajo. Todos los autores revisaron el manuscrito críticamente y participaron en la interpretación de los datos. Agradecemos a todas las participantes por su colaboración.

\section{Conflicto de intereses}

Los autores declaran no tener conflicto de intereses.

\section{Referencias}

Campbell, J. C. (2002). Health consequences of intimate partner violence. Lancet, 359, 1331-1336. doi:10.1016/ S0140-6736(02) 08336-8

Capaldi, D. M., Knoble, N. B., Shortt, J. W. y Kim, H. K. (2012). A Systematic Review of Risk Factors for Intimate Partner Violence. Partner Abuse, 3, 231-280. doi:10.1891/1946-6560.3.2.231 
Chen, P. H., Rovi, S., Vega, M., Jacobs, A. y Johnson, M. S. (2005). Screening for domestic violence in a predominantly Hispanic clinical setting. Family Practice, 22, 617623. doi:10.1093/fampra/cmi075

Cohen, L. R., Field, C., Campbell, A. N. y Hien, D. A. (2013). Intimate partner violence outcomes in women with PTSD and substance use: A secondary analysis of NIDA Clinical Trials Network "Women and Trauma» Multi-site study. Addictive Behaviors, 38, 2325-2332. doi:10.1016/j.addbeh.2013.03.006

Desmarais, S. L., Reeves, K. A, Nicholls, T. L., Telford, R. P. y Fiebert, M. S. (2012). Prevalence of Physical Violence in Intimate Relationships: Part 1. Rates of Male and Female Victimization. Partner Abuse, 3, 140-169.

Devries, K. M., Mak, J. Y., García-Moreno, C., Petzold, M., Child, J. C., Falder, G., ... Watts, C. H. (2013). Global health. The global prevalence of intimate partner violence against women. Science, 340, 1527-1528. doi:10.1126/ science. 1240937

Devries, K. M., Child, J. C., Bacchus, L. J., Mak, J., Falder, G., Graham, K., ... Heise, L. (2014). Intimate partner violence victimization and alcohol consumption in women: a systematic review and meta-analysis. Addiction (Abingdon, England), 109, 379-391. doi:10.1111/add.12393

El-Bassel, N., Gilbert, L., Wu, E., Go, H. y Hill, J. (2005). Relationship between drug abuse and intimate partner violence: a longitudinal study among women receiving methadone. American Journal of Public Health, 95, 465470. doi:10.2105/AJPH.2003.023200

El-Bassel, N., Gilbert, L., Witte, S., Wu, E. y Chang, M. (2011). Intimate partner violence and HIV among drug-involved women: Contexts linking these two epidemics-challenges and implications for prevention and treatment. Substance Use E Misuse, 46, 295-306. doi:10.3 109/10826084.2011.523296.

Feingold, A., Washburn, I. J., Tiberio, S. S. y Capaldi, D. M. (2015). Changes in the Association of Heavy Drinking and Drug Use with Intimate Partner Violence in Early Adulthood. Journal of Family Violence, 30, 27-34. doi:10.1007/s10896-014-9658-6

Gilbert, L., El-Bassel, N., Rajah, V., Foleno, A., Fontdevila, J., Frye, V. y Richman, B. L. (2000). The converging epidemics of mood-altering-drug use, HIV, HCV, and partner violence: a conundrum for methadone maintenance treatment. Mount Sinai Journal of Medicine, 67, 452-464.

Gilchrist, G., Blázquez, A. y Torrens, M. (2012). Exploring the relationship between intimate partner violence, childhood abuse and psychiatric disorders among female drug users in Barcelona. Advances in Dual Diagnosis, 5, 46-58. doi:10.1108/17570971211241895

Hegarty, K., Sheehan, M. y Schonfeld, C. (1999). A multidimensional definition of partner abuse: development and preliminary validation of the Composite Abuse Scale. Journal of Family Violence, 14, 399-414.
Hussain, N., Sprague, S., Madden, K., Hussain, F. N., Pindiprolu, B. y Bhandari, M. (2015). A comparison of the types of screening tool administration methods used for the detection of intimate partner violence: a systematic review and meta-analysis. Trauma, Violence $\mathcal{E}$ Abuse, 16, 60-69. doi:10.1177/1524838013515759

Kraanen, F. L., Vedel, E., Scholing, A. y Emmelkamp, P. M. G. (2014). Prediction of intimate partner violence by type of substance use disorder. Journal of Substance Abuse Treatment, 46, 532-539. doi:10.1016/j.jsat.2013.10.010

Langhinrichsen-Rohling, J., McCullars, A. y Misra, T. (2012). Motivations for Men and Women's Intimate Partner Violence Perpetration: A Comprehensive Review. Partner Abuse, 3, 1-33. doi:10.1891/1946-6560.3.4.e10

Leadley, K., Clark, C. y Caetano R. (2010). Couples' drinking patterns,intimate partner violence, and alcohol-related partnership problems. Journal Substance Abuse, 11, 253-263.

Ministerio de Sanidad y Políticas Sociales. (2015). Macroencuesta Violencia contra la Mujer 2015. Recuperado de: http://www.msssi.gob.es/gabinetePrensa/notaPrensa/pdf/30.03300315160154508.pdf

Reingle, J. M., Jennings, W. G., Connell, N. M., Businelle, M. S. y Chartier, K. (2014). On the pervasiveness of event-specific alcohol use, general substance use, and mental health problems as risk factors for intimate partner violence. Journal of Interpersonal Violence, 29, 29512970. doi:10.1177/0886260514527172

Sherin, K. M., Sinacore, J. M., Li, X. Q., Zitter, R. E. y Shakil, A. (1998). HITS: a short domestic violence screening tool for use in a family practice setting. Family Medicine, 30, 508-512.

Smith, P.H., Homish, G.G., Leonard, K.E. y Cornelius, J.R. (2012). Intimate partner violence and specific substance use disorders: findings from the National Epidemiologic Survey on Alcohol and Related Conditions. Psychology of Addictive Behaviors, 26, 236-245. doi:10.1037/a0024855.

Stöckl, H., Devries, K., Rotstein, A., Abrahams, N., Campbell, J., Watts, C. y Moreno, C. G. (2013). The global prevalence of intimate partner homicide: a systematic review. Lancet, 382, 859-865. doi:10.1016/ S0140-6736(13)61030-2

Tirado-Muñoz, J., Gilchrist, G., Lligoña, E., Gilbert, L. y Torrens, M. (2015). A group intervention to reduce intimate partner violence among female drug users. Results from a randomized controlled pilot trial in a community substance-abuse center. Adicciones, 27, 168-178.

Wagner, K. D., Hudson, S. M., Latka, M. H., Strathdee, S. A., Thiede, H., MacKesy-Amiti, M. E. y Garfein, R. S. (2009). The effect of intimate partner violence on receptive syringe sharing among young female injection drug users: An analysis of mediation effects. AIDS and Behavior, 13, 217-224. doi:10.1007/s10461-007-9309-5

Weaver, T. L., Gilbert, L., El-Bassel, N., Resnick, H. S. y Noursi, S. (2015). Identifying and intervening with 
substance-using women exposed to intimate partner violence: phenomenology, comorbidities, and integrated approaches within primary care and other agency settings. Journal of Women's Health (Larchmt), 24, 51-56. doi:10.1089/jwh.2014.4866

World Health Organization. (2013). Responding to intimate partner violence and sexual violence against women: WHO clinical and policy guidelines. Recuperado de: http://apps.who.int/iris/bitstre am/10665/85240/1/9789241548595_eng.pdf?ua=1 